\title{
Optimization of Spreading Code using Modified Differential Evolution for Wireless Communication
}

Katyayani Kashyap ( $\nabla$ kashyap.katyayani@gmail.com )

Amity University Rajasthan https://orcid.org/0000-0002-2817-6265

\section{Sunil Pathak}

Amity University Rajasthan: Amity University - Jaipur Campus

\section{Narender Yadav}

Manipal University - Jaipur Campus

\section{Research Article}

Keywords: Spreading Code, Differential Evolution, Optimization, Wireless Communication, Logistic map, Wavelet Transform

Posted Date: June 29th, 2021

DOI: https://doi.org/10.21203/rs.3.rs-379740/v1

License: (c) (1) This work is licensed under a Creative Commons Attribution 4.0 International License. Read Full License

Version of Record: A version of this preprint was published at Wireless Personal Communications on August 17th, 2021. See the published version at https://doi.org/10.1007/s11277-021-08949-4. 


\title{
Optimization of Spreading Code using Modified Differential Evolution for Wireless Communication
}

\author{
Katyayani Kashyap*, \\ Research Scholar, Amity University, Rajasthan, India \\ kashyap.katyanyani@gmail.com. \\ ${ }^{2}$ Dr. Sunil Pathak \\ Associate Professor \& Head, Department of CSE \\ Amity University Jaipur, Rajasthan, India. \\ sunilpath@gmail.com \\ ${ }^{3}$ Dr. Narender Yadav \\ Associate Professor, Manipal University, Jaipur, India \\ narensinghyadav@yahoo.com
}

\section{Abstract:}

Spread spectrum linked to optimization techniques play a very important role in today's world. Optimization gives the best one among all the solutions and the spread spectrum is used to minimize distortion, hence optimized spreading code is very important in wireless communication. This study shows a comparison of two recent modified techniques of DE with the help of engineering design problems and application of that modified DE (considering the better one) in wireless communication to optimize logistic map based spreading code. A comparative study of properties of both optimized and non-optimized spreading code is also discussed in this paper. The performance of the optimized spreading code is also evaluated with the help of bit error rate (BER) by applying it in static and dynamic direct sequence spread spectrum system (DSSS). The performance of the proposed technique (optimized dynamic logistic map code based DSSS) displays better results than non-optimized spreading code and orthogonal spreading code considering BER. Therefore, the proposed method can be efficiently applied in the wireless communication system.

Keywords: Spreading Code, Differential Evolution, Optimization, Wireless Communication, Logistic map, Wavelet Transform. 


\section{Introduction}

The meaning of the spread spectrum in wireless communication means signal bandwidth expansion by multiplying the message signal with another signal called spreading signal (PN sequence). Starting from the military applications, in the current and also in the next generation of wireless communication techniques, spread spectrum plays a vital role [1], [2]. Using chaotic code (Logistic map code) in spread spectrum communication shows powerful properties like interference mitigation, low probability of intercept, less bit error rate (BER), etc. over the conventional methods like Gold code, PN code, Kasami code, Walsh code, etc. Not only that, the results can be enhanced by applying optimization in chaotic code. Optimization is a very important topic because it helps to find the best solution from the number of solutions. Hence optimized chaotic code plays a very important role in wireless communication techniques [3], [4]. Considering optimization, Differential evolution (DE) is an important evolutionary technique, enhances its parameters such as, initialization of population, mutation, crossover, etc. to resolve realistic optimization issues also in wireless communication [5], [6]. To solve global optimization issues, DE plays a very important role, which is a well-known evolutionary algorithm based on population and it is proposed [7]. Literature reflects that DE shows high-quality performance in application with different optimization problems [8]. Also various enhance methods of DE by modifying its parameters show a very good performance comparing to different recent optimization techniques [9].

This paper shows a comparative study of two recent modified techniques of DE with the help of engineering design problems and application of that modified DE (considering the better one) in wireless communication to optimize logistic map based spreading code. In a comparative study of DE, modification of DE is done with the help of wavelet transform [9] and exponential scale factor. A comparative study of properties of both optimized and non-optimized spreading code is also discussed in this paper. The performance of the optimized spreading code is also evaluated with the help of bit error rate (BER) by applying it in static and dynamic direct sequence spread spectrum system (DSSS). The performance of the proposed technique (optimized logistic map code based DSSS) displays better results considering BER. Hence the proposed method can be efficiently applied in the wireless communication system.

The paper is prearranged as shown in the following; the literature review is discussed in Section 2. The background is in Section 3. The proposed method is explicated in Section 4. Parameter settings and experimental outcomes are illustrated in Section 5. Section 6 presents the conclusion and references are in section 7 .

\section{Literature Review}

The modification of the initialization of population along with the mutation rate is done in [9] using logistic map and wavelet transformation in DE respectively which increases the convergence rate and accordingly, after which test is done on various benchmarks problems. Thereafter, the results are compared with the state of the art algorithms along with different real-time none linear engineering problems to obtain their performance.

An inflationary differential evolution based on multipopulation adaption is proposed in [6] which combines the local search mechanisms of monotonic basin hopping with the basic differential evolution as a result of which the differential evolution parameters namely $\mathrm{CR}$ and $\mathrm{F}$ get automatically adapted with the size of the local restart bubble as well as the number of local restarts of monotonic basin hopping. The aforesaid algorithm a simple as well as effective mechanism to avoid multiple detections of the same local minima and it allows the algorithm to decide as to whether to start a local research. The algorithm has been tested over more than 50 test functions.

A comparison is made by in [5] between the performance of others spreading code in terms of Bit Error Rate, Cross corelation and average interference which is presented by comparing the generation of optimum chaotic spreading codes by using genetic algorithm.

The derivation of generation of binary sequence from chaotic sequences over $\mathrm{Z} 4$ is proposed in [3] and the six chaotic situation which are considered in [3] are Tent Map, Logistic map, Quadratic Map, Cubic Map, and Bernoulli Map and using this equations generation of sequence over $\mathrm{Z} 4$ is obtained and the same are converted by using polynomial mapping to binary sequence. Moreover, for cross co-relation and linear-complexity properties, the segments of sequences of different lengths are tested and accordingly it is found that some segments of different lengths have got good co-relations and linear-complexity properties. As compared to the Gold sequences and Kasami sequences, the Bit Error Rate performance in the DS-CDMA communications system is found to be better by using this binary sequences.

This article presentation is made on a design of a dynamic chaotic spreading sequence to apply them in a Direct Sequence Spread Spectrum (DSSS) based system along with the considerations of wireless channels (Rayleigh and Rician) in [1]. Moreover, a presentation is made after a comparison of a linear and none linear channels with static chaotic sequence in term of a bit error rate, computational time, mutual information and signal power for faded channel, evaluations of performance is done taking into consideration of different modulation schemes, which finally dictates the efficiency of generated code. A comparison of the performance of the generated dynamic logistic map-based sequence is done to that of what is obtained from the gold code under equivalent conditions.

Presentation of the detailed history, characteristics strengths, variants and weaknesses of DE are made in [4]. Identification of seven broad areas are made as different domains of application of DE in wireless communications it was observed that the two major areas where the DE is applied are the coverage area maximization and energy consumption minimization. Quality of service, updating mechanism are the other areas where candidate positions learn from a large diversified search region, security and related filed applications. 
The implementation of ultra-wideband (UWB) communication systems with eight transmitting and receiving ring in antenna arrays are done in [10], to test the bit error rate and capacity performance. Calculation of the impulse response of the system is done by using the ray-tracing technique to compute any given indoor wireless environment. To find the excitation current and also to feed the line length of each antenna to form the appropriate beam pattern which can reduce the bit error rate and which can increase the channel capacity and receiving energy. It is seen by $\mathrm{C}$. Chiu that in both of sight and non-line, the SADDE had better results. Not only are that other different methods of modification of DE and also application in various fields also reflected in [11-18].

\section{Background}

In today's world, the spectrum will be a scarce commodity; its utilization shall be of utmost importance. Hence spread spectrum linked to optimization techniques plays a vital role in today's world [19]. Works of literature make it clear that PN and Gold codes are limited to fixed sequence lengths with a system configuration. Also, flexibility is less because we cannot generate multiple numbers of sequences for the same sequence length. Usually, to generate the PN sequence, certain sum-store blocks and LFSR (linear feedback shift register) are used. Also, to generate Gold code LFSR is used because the generation of Gold code is done by exclusive-OR of two PN sequences, hence for both PN and Gold code, a physical structure is needed which consumes more power with less flexibility [1], [20-22]. There are deviations in the propagation medium in fading conditions. In that case, a flexible spreading code is more suitable than a fixed length one. A logistic map based signal is the sensitive dependence on the initial condition and these properties have increased the interest in using chaos in many fields of Science and Telecommunications [23]. Chaos codes are dynamically formulated combinations that have been established to be useful for a host of scenarios in wireless communication. The generation of chaotic code is very simple. Even for very long sequences only a few functions and parameters are needed. In addition, a huge number of diverse sequences can be generated just by changing its initial condition. Chaotic sequences are reproducible, correlated and dynamic, which can be very helpful in enhancing the security of transmission in communication. Again optimization is a technique by which we can find the best solution among all the solutions. Here, differential evolutionary algorithm is used as a stochastic algorithm [10], [24]. Stochastic algorithms are generally nature-inspired algorithms, they have flexible behavior to adapt to a changing environment [25]. Therefore, the primary focus of the work is to explore the possibility of improved performance that may be derived using modified Differential Evolution. Further, the deployment of modified $\mathrm{DE}$ in a chaotic system in wireless communication and other engineering applications. To optimize chaos code Differential Evolutionary Algorithm can be considered because it has random search nature, fewer parameter setting, valid for high dimensional complex problems and have high performances. Also, hybridization of stochastic algorithm is important to improve the Convergence Speed, to balance the Exploration (diversification) and Exploitation (intensification) process, to avoid trapping in local optima and to improve the quality of the solutions. Hence, the work explores the spin-offs that are likely to be obtained from optimized link oriented chaos system supported wireless techniques.

\section{The proposed method}

\subsection{Comparison of two different modified DE}

In this section at first two different modified DE are compared by applying it in engineering design problems. The difference between the modified DE is explained with the help of the flow diagrams as shown in Fig.1 and Fig.2.

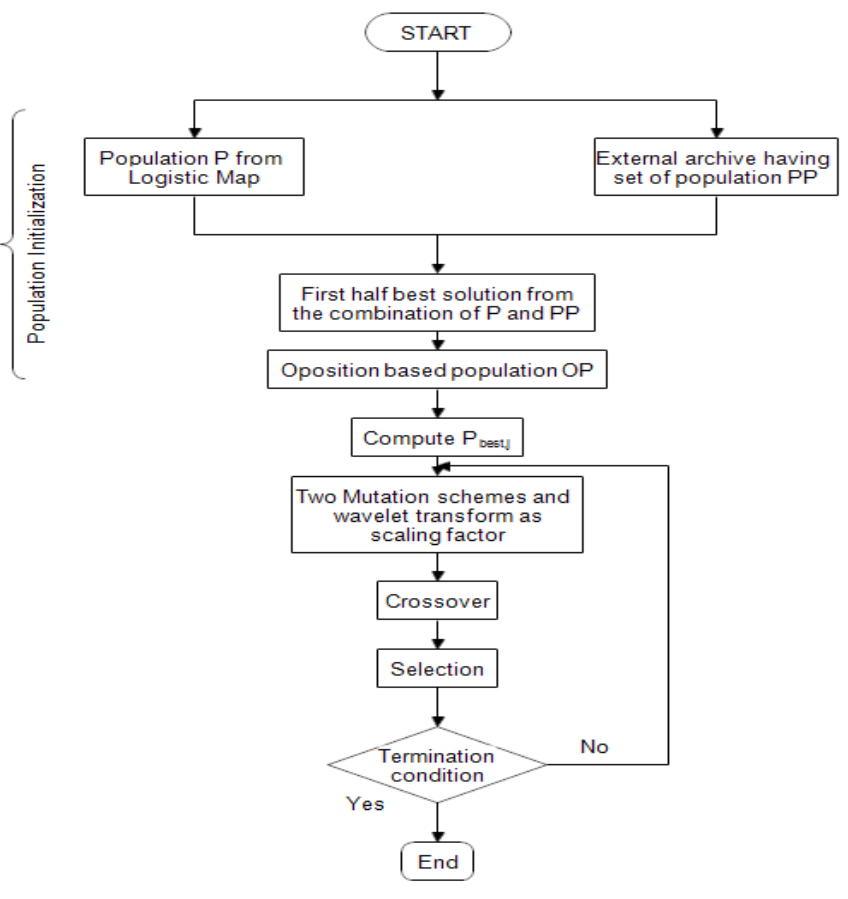

Fig. 1 Flow diagram of modified DE

Fig. 1 and Fig. 2 describe that initialization of the population is same in both the case but the mutation is varied as explained below.

In Fig. 1, two mutation schemes are used, DE/best /1/bin and $\mathrm{DE} / \mathrm{rand} / 1 / \mathrm{bin}$ which is explained below correspondingly with the help of mathematical terms [9].

$$
\begin{aligned}
& v_{\mathrm{i}}^{\mathrm{k}+1}=\mathrm{x}_{\text {best }}^{\mathrm{k}}+\mathrm{F}\left(\mathrm{x}_{\mathrm{i} 1}^{\mathrm{k}}-\mathrm{x}_{\mathrm{i} 2}^{\mathrm{k}}\right), \text { if } \delta<0 ; \\
& \mathrm{v}_{\mathrm{i}}^{\mathrm{k}+1}=\mathrm{x}_{\mathrm{i} 3}^{\mathrm{k}}+\mathrm{F}\left(\mathrm{x}_{\mathrm{i} 1}^{\mathrm{k}}-\mathrm{x}_{\mathrm{i} 2}^{\mathrm{k}}\right), \text { if } \delta \geq 0 .
\end{aligned}
$$

Here, $\mathrm{x}_{\text {best }}^{\mathrm{k}}=$ best solution; i1, i2, i3 are not equal and are different integer numbers having range $[1, \mathrm{M}] ; \delta=$ selection probability. Also, $\mathrm{F}$ (scale factor) is not constant andF $=\delta=\psi_{\mathrm{d}}(\varnothing)=\frac{1}{\sqrt{\mathrm{d}}} \psi\left(\frac{\emptyset}{\mathrm{d}}\right)$ 
Where, $\mathrm{d}=\mathrm{e}^{\ln (\lambda) \times\left(1-\frac{\mathrm{i}}{\mathrm{I}}\right)^{\zeta} \mathrm{wm}}+\ln (\lambda)$ and $\lambda=$ upper limit of $\mathrm{d}, \mathrm{i}=$ current iteration number, $\mathrm{I}=$ total iteration number, $\zeta_{\mathrm{wm}}$ $=$ shape parameter, is a monotonically increasing function.

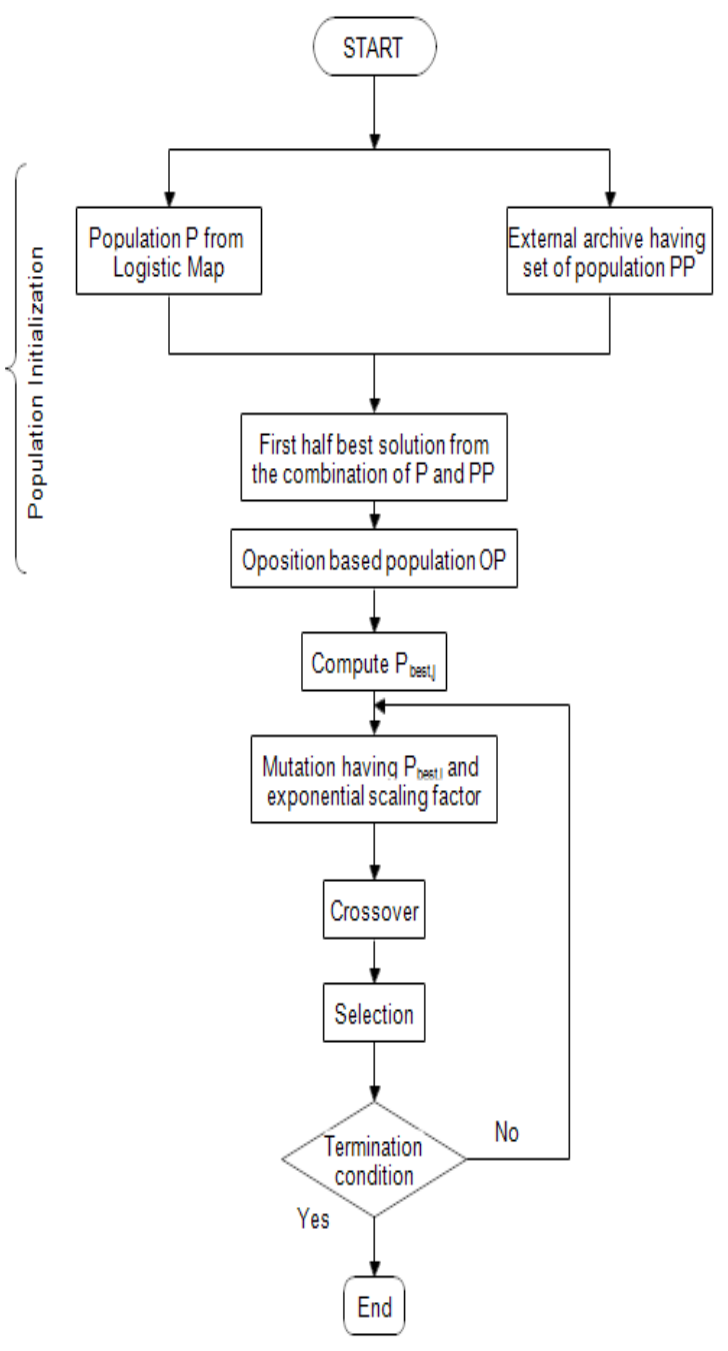

Fig. 2 Flow diagram of modified DE

In basic $\mathrm{DE}, \mathrm{F}$ the scaling factor, $\mathrm{CR}$ the crossover rate are constant values, but in modified DE from Fig. 2, F is replaced by taking exponential scaling factor $\mathrm{f}$ [26]. In DE, using scaling factor $(\mathrm{F})$ and crossover rate $(\mathrm{CR})$, exploration and exploitation are balanced. For greater value of $F$ and $C R$, in the search area, there is a high probability to skip the true solution. To overcome this drawback, the scale factor is replaced by an exponential scale factor as shown in equation (3). During the stage of initial iteration, the value of $\mathrm{f}$ will be high, which helps in the search area in exploration, after drop of some iterations, exponentially the value of $f$ will decrease, hence with reduced step size, the solution is now to move and which enhances the exploitation skill of the algorithm. By using this approach, the proper equilibrium between exploration and exploitation skills of the algorithm can be upheld.

$$
\text { Where, } \mathrm{f}=1 / \exp (1-(\mathrm{K}-\mathrm{i}) / \mathrm{K}) .
$$

Here, $\mathrm{K}$ represents maximum iteration number, i represents the current iteration.

\subsection{Optimization of spreading code using modified $D E$}

The flow diagram of the optimized spreading code is as shown the Fig. 3

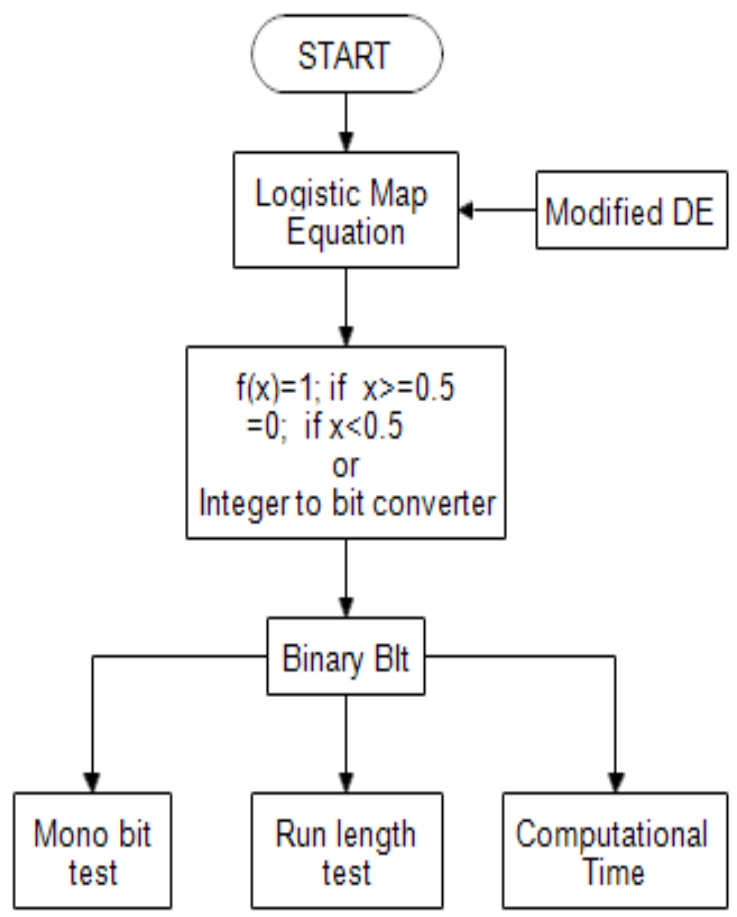

Fig. 3 Flow diagram to generate an optimized binary sequence

As wavelet-based DE shows better results as explained in section 5 than exponential based DE, Logistic map based spreading sequences are optimized using the wavelet-based DE as shown in the flow diagram in Fig.3. Binary bits are generated using the thresholding method (method 1) and integer to bit conversion method (method 2) [9]. Properties (Mono bit test, run-length test, computational time) of optimized generated bits are compared with the nonoptimized one [1] as shown in the Table as shown in section 5.

4.3. Application of optimized spreading code in static and dynamic Direct Sequence Spread Spectrum System to calculate BER (Bit Error Rate) for signal detection. 


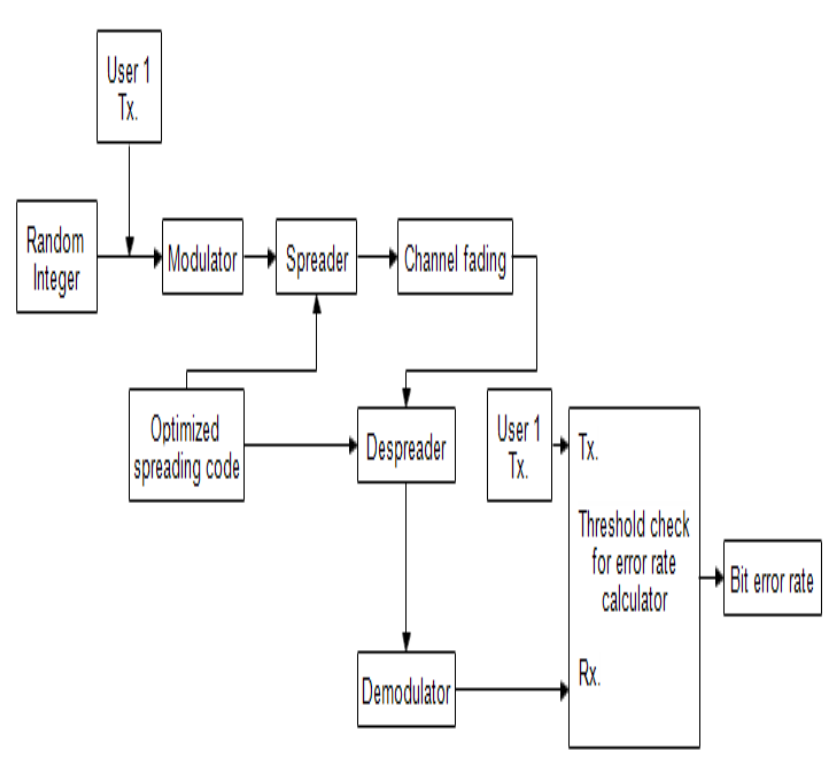

Fig. 4 System model of static DSSS

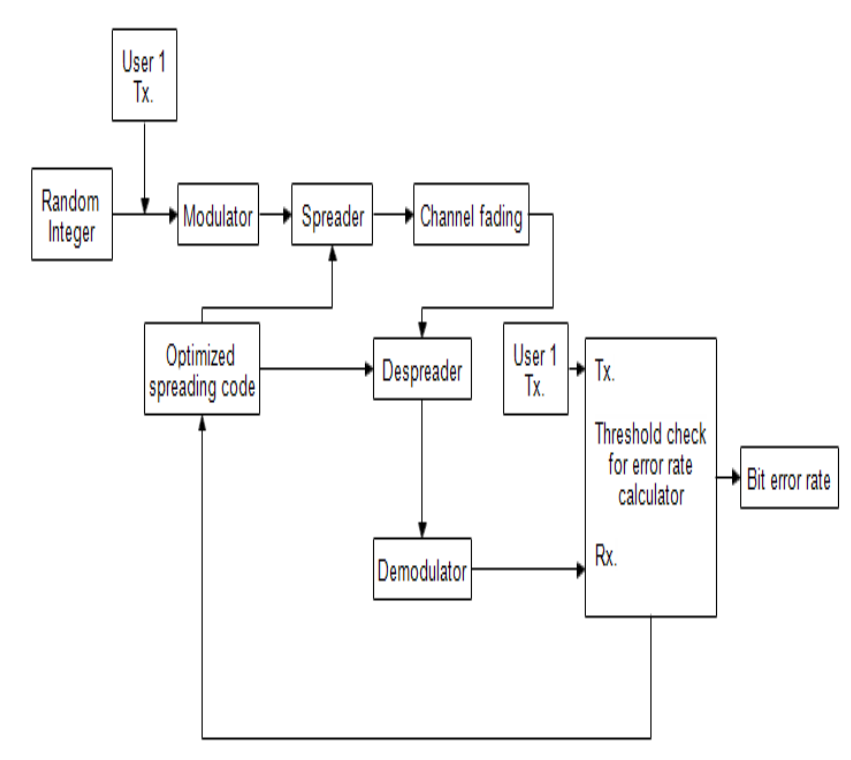

Fig.5 System model of dynamic DSSS

Fig. 4 and Fig. 5 shows the static and dynamic direct sequence spread spectrum (DSSS) respectively. Fig. 4 displays the static DSSS with an optimized logistic map based spreading code and Fig. 5 shows that the dynamic DSSS with optimized logistic map based spreading code. The basic fundamental of DSSS is that binary bits are speeded over the channel bandwidth. First, from a random source data is generated. It consists of a series of ones and zeros. In modulation data bits are mapped into symbol vector. The modulation scheme used in this work is BPSK [1]. The modulated data is spreaded by an optimized logistic map based spreading code in the transmitter section in Fig. 4. After passing through the nonlinear channel, the received signal is de spreader using the same optimized spreading code and then demodulated using the BPSK demodulator. The word dynamic is used in Fig. 5 because the optimized spreading code is not fixed, the length is changed with the BER (bit error rate) value obtained comparing to the threshold value.

Mathematical equations of the proposed system are explained in the following.

1) Received signal:

$k_{m, n}(b, \theta)=f_{1}(b(n), d(n)) \cdot S_{n} * H_{m, n}(b, \theta)+N$

Where

- $f_{1}(b(n), d(n))$ is a modulation process,

- $\quad P(b, \theta)=$ Rayleigh $h_{m, n}\left(1+b+b^{2}+\cos \theta\right)$ is the channel matrix for $b$ sample value and $\theta$ is the phase with nonlinear terms,

- $G_{n}=\operatorname{Step}\left(q_{n}\right)$ with $q_{n+1}=r * q_{n}\left(1-q_{n}\right)$ is a optimized logistic map-based sequence with $r=$ 3.582. (Optimization algorithm is wavelet-based differential evolution)

2) In recovery,

While (BER <Threshold)

- $\quad$ Update $G_{n}$ and repeat $k$ as in step 1 .

\section{Parameter Settings and Experimental Results}

To compare both the modified algorithms of $\mathrm{DE}$ and to analysis, the efficiency as explained in section 4, the important benchmark functions used are Perm function, Levy function, Schwefel function, Sum of different powers function, Trid function, Griewank function, Sphere function, Ackley function, Rastrigin function, and Zakharov [9]. Table 1 shows the parameter setting. The comparative outcomes are as shown in Table 2, f1 is Gas transmission compressor design, $\mathrm{f} 2$ is Optimal capacity of gas production facilities and $\mathrm{f} 3$ is the Design of a gear train[9]. From Table 2 it is clear that waveletbased DE gives better results than exponential DE considering real-time engineering design problems [9], [27]. Table 3 shows the number of function evaluations for different algorithms, and it is clear from table 3 that wavelet-based DE converges more quickly than exponential based DE. Further, a comparative study is done for optimized and non-optimized binary sequences considering the properties of generated bits as shown in Table 4 . Table 4 shows that, when we consider method 1 (thresholding) and method 2 (integer to bit conversion) for both optimized and non-optimized bits, method 2 gives better results than method 1 for both mono bit test and run-length test. But computational time required is more in case of the thresholding method. Also when we consider optimized and non-optimized binary sequences, it is clear from Table 4 that optimized binary sequences show better mono bit test and run-length test properties. Hence optimized version of method 1 is used in a direct sequence spread spectrum system (DSSS). Fig. 6 shows the bit error rate (BER) curves for static non-optimized, static optimized and dynamic optimized DSSS. From Fig. 6 we can say that static optimization gives better results than static non-optimization because the BER curve for static optimization is more close to 
the theoretical value. Also when we consider static and dynamic, the dynamic is more close to the theoretical one. Hence we can say from the analysis of the result that dynamic optimization gives better results than static optimization or static non-optimization. Fig. 7 shows the bit error rate (BER) curves for orthogonal, static optimized and dynamic optimized spreading sequence and it is clear from Fig. 7 that dynamic optimization is more closer to the theoretical value comparing to the remaining BER curves means gives the better result. But when we compare static optimization and orthogonal, orthogonal gives the better result than the static optimization. Fig. 8 explains the BER curves for optimized spreading sequence for DPSK, QPSK and QPSK modulation and the BER curves describe that BPSK and QPSK almost gives the same BER and better than DPSK modulation. Fig. 9, Fig. 10 and Fig. 11 show the convergence speed graph of function $\mathrm{f} 1, \mathrm{f} 2$, and $\mathrm{f} 3$ for different algorithms.

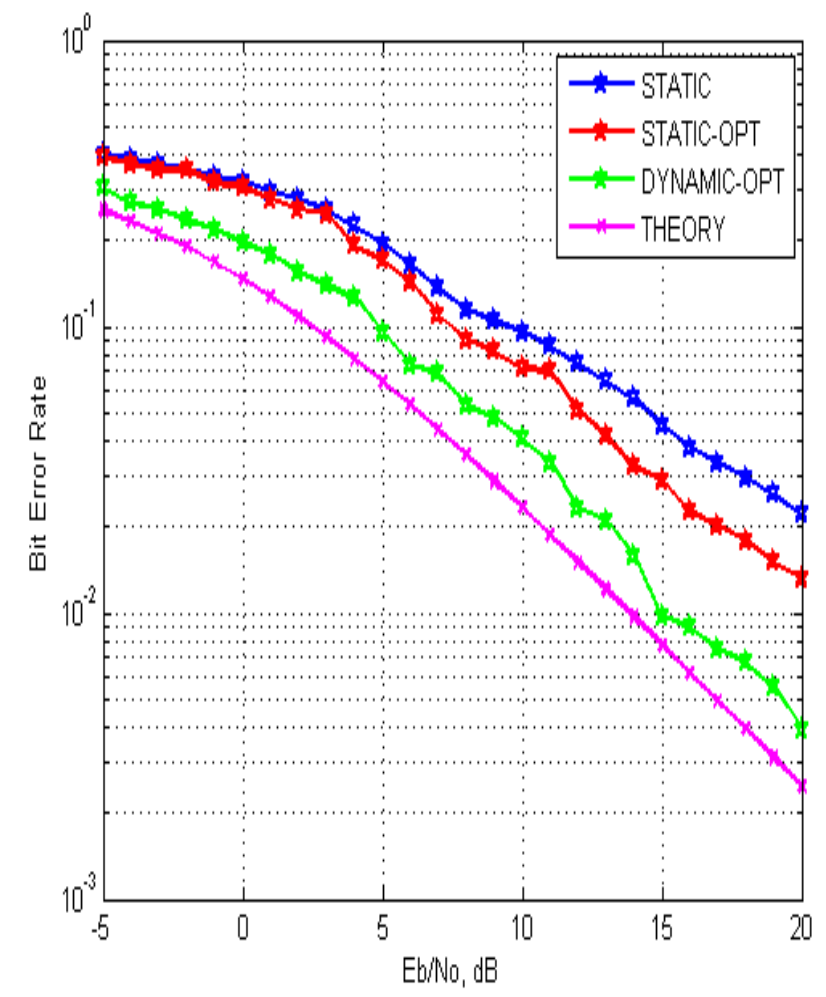

Fig. 6: BER curves for static non-optimized, static optimized and dynamic optimized DSSS

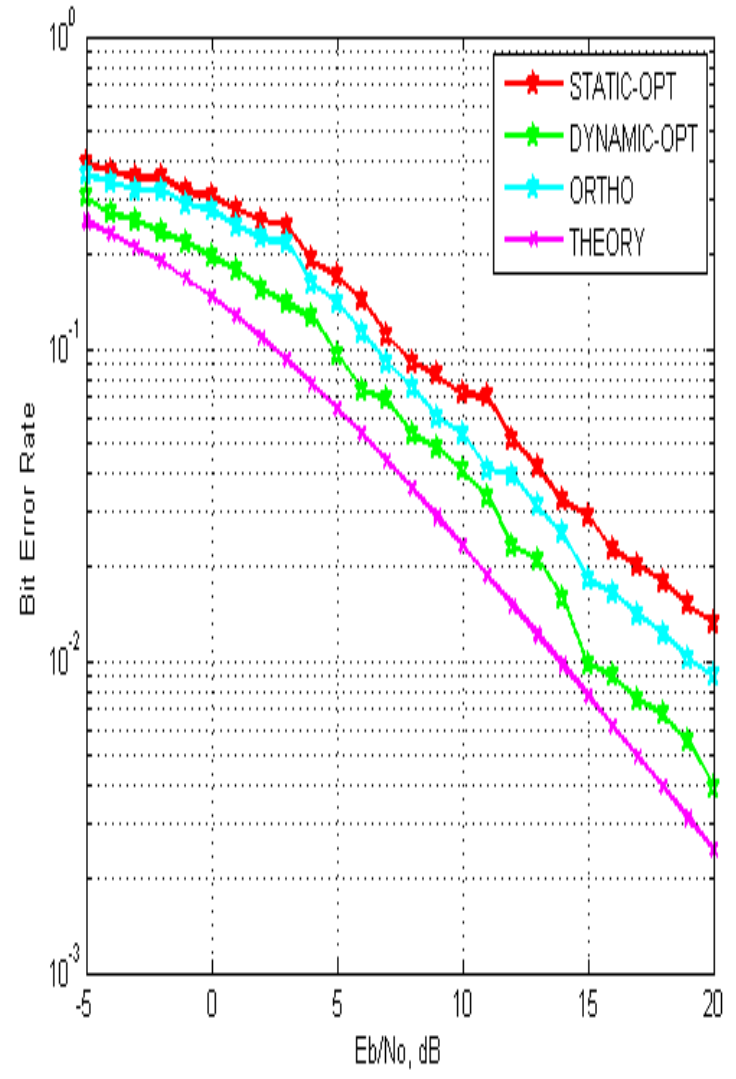

Fig. 7: BER curves for orthogonal, static optimized and dynamic optimized spreading sequence

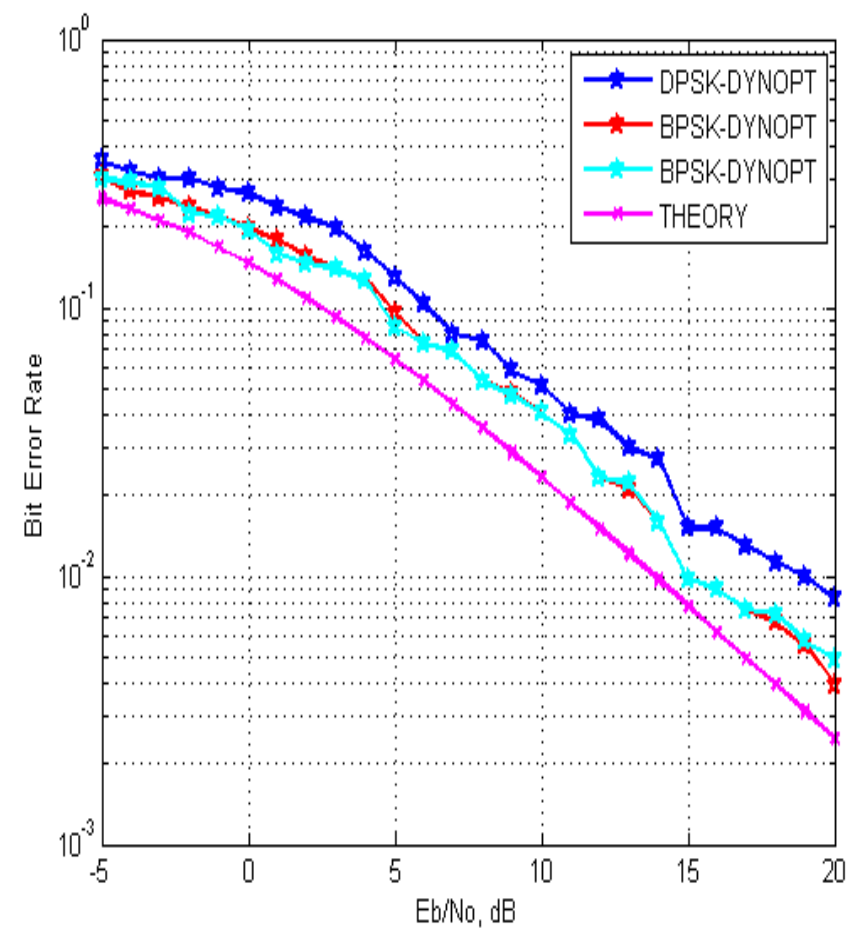

Fig. 8: BER curves for optimized spreading sequence For DPSK, QPSK and QPSK modulation 


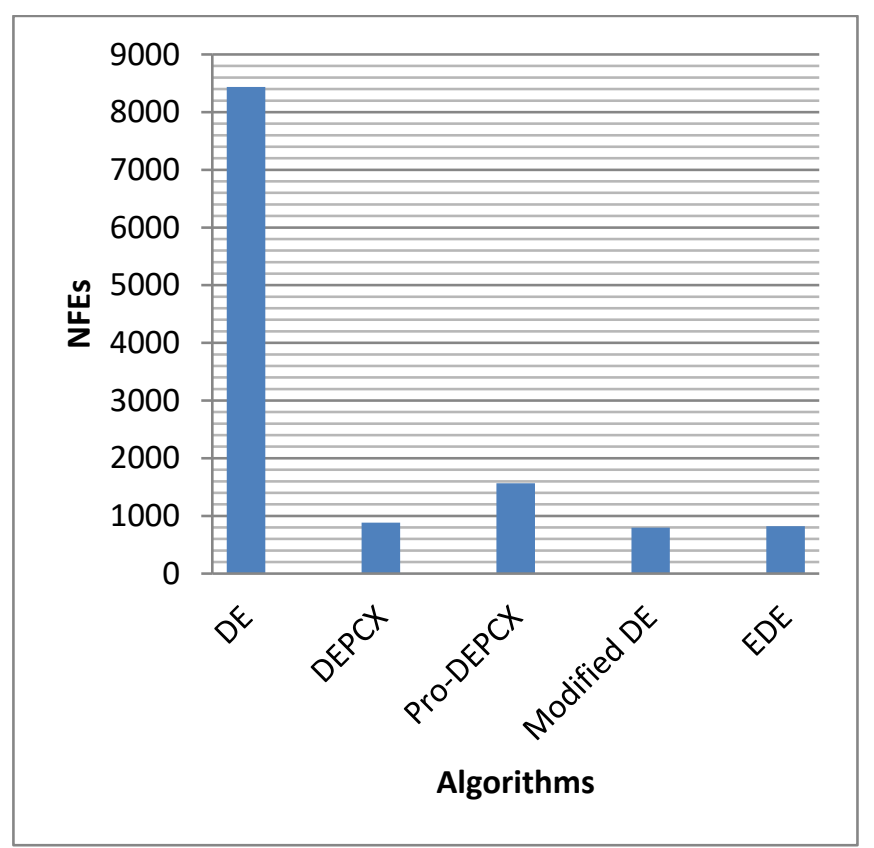

Fig.9 Number of function evaluation for function $\mathrm{f} 1$

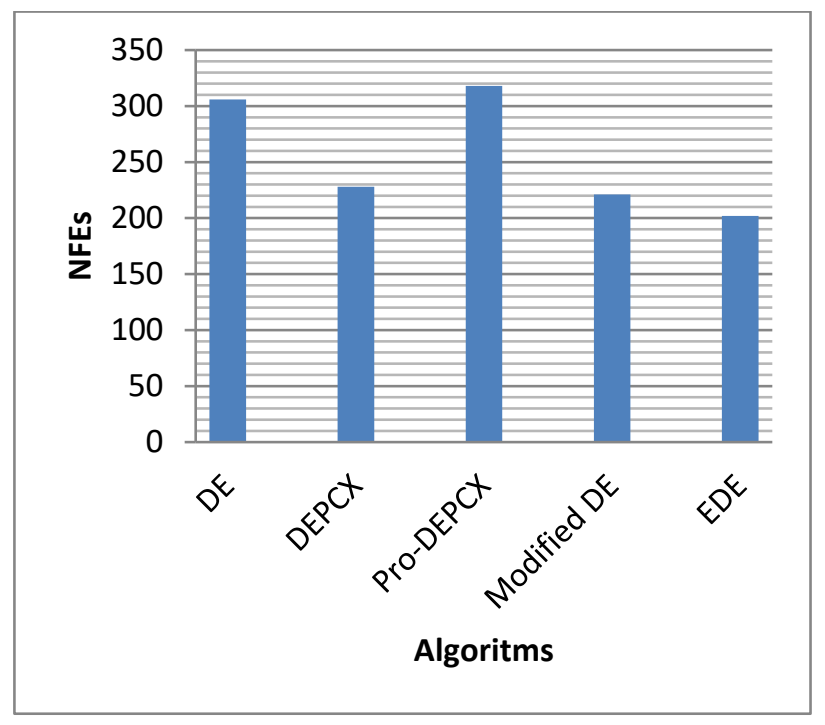

Fig.10 Number of function evaluation for function $\mathrm{f} 2$

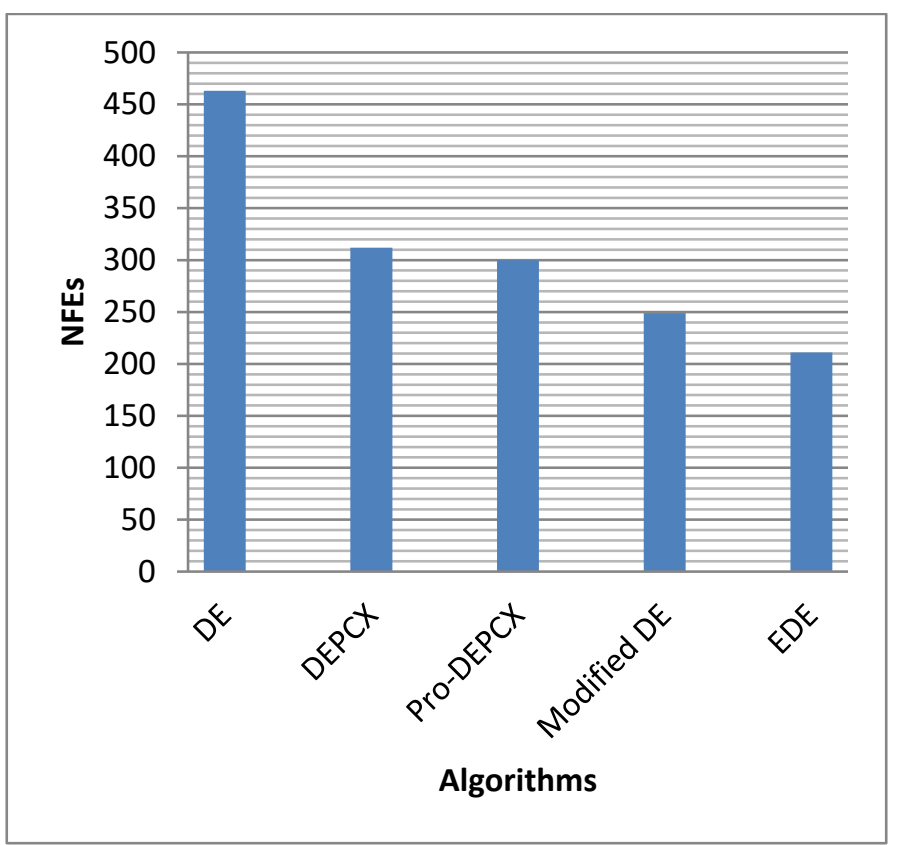

Fig.11 Number of function evaluation for function $\mathrm{f} 3$

Table 1: Simulation Parameter

\begin{tabular}{|l|l|l|}
\hline $\begin{array}{l}\text { Sl. } \\
\text { No. }\end{array}$ & Item & Description \\
\hline 1 & Modulation type & BPSK, QPSK, DPSK \\
\hline 2 & Data block size & Between 1000 to 10,000 \\
\hline 3 & Logistic map code & $\begin{array}{l}\text { Static, Static optimized, } \\
\text { Dynamic optimized, } \\
\text { Orthogonal }\end{array}$ \\
\hline 4 & $\begin{array}{l}\text { Value of r in the } \\
\text { logistic map }\end{array}$ & $\begin{array}{l}3.582 \\
\text { AWGN, } \\
\text { (nonlinear) }\end{array}$ \\
\hline 5 & $\begin{array}{l}\text { Channel type } \\
\text { sequence length }\end{array}$ & At least 10 \\
\hline 7 & $\begin{array}{l}\text { Modified DE } \\
\text { Wavelet-based DE }\end{array}$ \\
\hline
\end{tabular}


Table 2: Comparative study of Logistic Map and Wavelet-based DE using real-time optimization function.

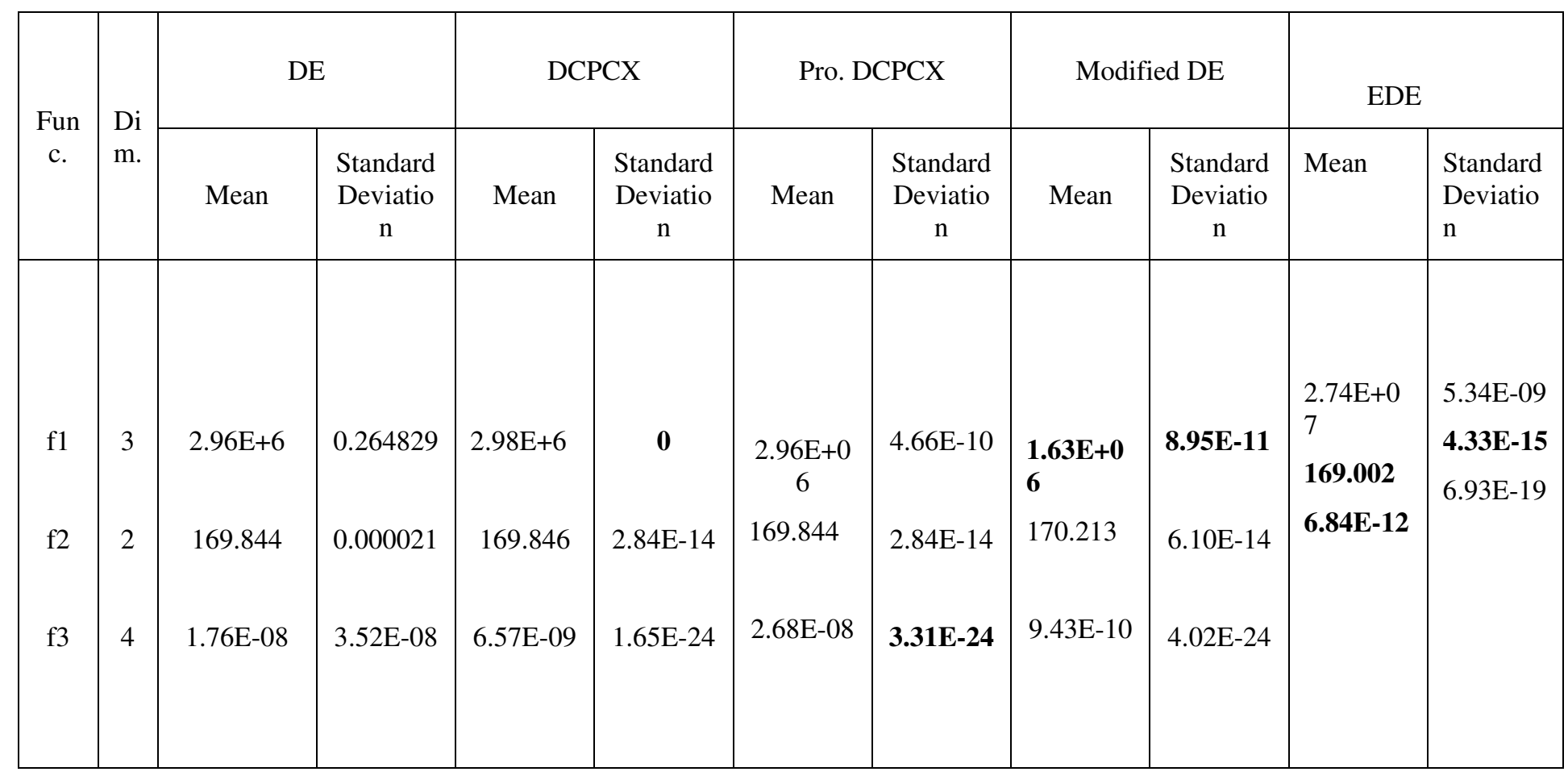

Table 3. Number of function evaluation (NFE) of real-time function Evaluation for different algorithms

\begin{tabular}{|c|c|c|c|c|c|c|}
\hline Func. & Dim & NFE (DE) & NFE(DEPCX) & $\begin{array}{l}\text { NFE (Pro } \\
\text { DEPCX) }\end{array}$ & Modified DE & EDE \\
\hline F1 & 3 & 8438 & 882 & 1566 & $\mathbf{7 9 8}$ & 820 \\
\hline F2 & 2 & 306 & 228 & 318 & 221 & $\mathbf{2 0 2}$ \\
\hline F3 & 4 & 463 & 312 & 300 & 249 & $\mathbf{2 1 1}$ \\
\hline
\end{tabular}

Table 4: Comparative study of Properties of the generated bit sequence

\begin{tabular}{|c|c|c|c|c|}
\hline \multirow{2}{*}{ Test } & \multicolumn{2}{|c|}{ Properties of non-optimized generated bit } & \multicolumn{2}{c|}{ Properties of optimized generated bit } \\
\cline { 2 - 5 } & Method-1 & Method-2 & Method-3 & Method-4 \\
\hline Mono bit Test & $\begin{array}{c}\text { No. of Zeros=66 } \\
\text { No. of Ones=34 } \\
\text { Out of } 100 \text { bits }\end{array}$ & $\begin{array}{c}\text { No. of Zeros=41 } \\
\text { No. of Ones=59 } \\
\text { Out of } 100 \text { bits }\end{array}$ & $\begin{array}{c}\text { No. of Zeros=62 } \\
\text { No. of Ones=38 } \\
\text { Out of } 100 \text { bits }\end{array}$ & $\begin{array}{c}\text { No. of Zeros=43 of Ones=57 } \\
\text { Out of } 100 \text { bits }\end{array}$ \\
\hline Run Length test & Run is 5 out of 20 bits & Run is 5 out of 20 bits & Run is 6 out of 20 bits & Run is 7 out of 20 bits \\
\hline Computational & CT is 1.02 seconds & CT is 1.94 seconds & CT is 1.38 seconds & CT is 2.11 seconds \\
\hline
\end{tabular}




\section{Conclusion}

Differential evolution (DE) with enhanced parameters can play a vital role to solve the realistic optimization problems and application in wireless communication techniques. To optimize chaos code differential evolutionary algorithm can be considered because it has the property of random search, requires only fewer parameters setting, high performance and valid to complex optimization problems. Also, hybridization of stochastic algorithm is important because, to improve the convergence speed, to balance the exploration (diversification) and exploitation (intensification) process, to avoid trapping in local optima and to improve the quality of the solutions. From the outcomes of the proposed system, it is clear that the optimized chaos code using modified DE can be used as a spreading code which gives better results than non-optimized spreading code and orthogonal spreading code. Hence, the work explores the spin-offs that can be obtained from optimized link oriented chaos system supported wireless techniques.

\section{Declaration}

*Funding (information that explains whether and by whom the research was supported): None

*Conflicts of interest/Competing interests : None

*Availability of data and material (data transparency) : All required data are provided in the tables.

* Code availability (software application or custom code) : Explained with the help of flow diagram.

\section{References}

[1] K. Kashyap and T. Sharma, "Dynamic Logistic MapBased Spread Spectrum Modulation in Wireless Channels", International Journal of Applied Evolutionary Computation, vol. 9, no. 2, pp. 52-65, 2018. Available: 10.4018/ijaec.2018040105.

[2] D. Tse and P. Viswanath, "Fundamentals of Wireless Communications", Cambridge, $2^{\text {nd }}$. Ed., 2005.

[3] M. V. Mandi, H. K.N and M. R, "Performance Evaluation of DS-CDMA System Using Chaotic Binary Sequences", Computer Applications: An International Journal, vol. 2, no. 1, pp. 37-52, 2015. Available: 10.5121/caij.2015.2104.

[4] H. Okagbue, M. Adamu and T. Anake, "Differential Evolution in Wireless Communications: A Review", International Journal of Online and Biomedical Engineering (iJOE), vol. 15, no. 11, p. 29, 2019. Available: 10.3991/ijoe.v15i11.10651.

[5] U. Rastogi, S. Anuradha, R. Shekar, S. Singh and P. Sri Hari Rao, "Optimal Chaotic Sequences for DSCDMA Using Genetic Algorithm", IEEE WiSPNET 2017, pp. 900-904.

[6] M. Di Carlo, M. Vasile and E. Minisci, "Adaptive multi-population inflationary differential evolution", Soft Computing, $2019 . \quad$ Available: 10.1007/s00500-019-04154-5.
[7] K.V. Price, R. M. Storn and J. A. Lampinen, "Differential evolution: apractical approach to global optimization Natural Computing" Series. Springer, Berlin, 2005.

[8] S. Das and P. Suganthan, "Differential Evolution: A Survey of the State-of-the-Art", IEEE Transactions on Evolutionary Computation, vol. 15, no. 1, pp. 431, 2011. Available: 10.1109/tevc.2010.2059031.

[9] K. Kashyap, T. Sharma and J. Rajpurohit, "Logistic map and wavelet transform based differential evolution", International Journal of System Assurance Engineering and Management, 2019. Available: 10.1007/s13198-019-00920-8.

[10] C. Chiu, Y. Tong and Y. Cheng, "Comparison of selfadaptive dynamic differential evolution and particle swarm optimization for smart antennas in wireless communication", International Journal of Communication Systems, vol. 32, no. 9, p. e3941, 2019. Available: 10.1002/dac.3941.

[11] R. Barpanda, A. Turuk and B. Sahoo, "QoS aware routing and wavelength allocation in optical burst switching networks using differential evolution optimization", Digital Communications and Networks, vol. 4, no. 1, pp. 3-12, 2018. Available: 10.1016/j.dcan.2017.09.002.

[12] T. Eltaeib and A. Mahmood, "Differential Evolution: A Survey and Analysis", Applied Sciences, vol. 8, no. 10, p. 1945, 2018. Available: 10.3390/app8101945.

[13]L. Cui et al., "Differential Evolution Algorithm With Tracking Mechanism and Backtracking Mechanism", IEEE Access, vol. 6, pp. 44252-44267, 2018. Available: 10.1109/access.2018.2864324.

[14]S. Veisee and S. Asadi, "A Modified Unequally Spaced Array Antenna Synthesis Method for Side Lobe Reduction", Radioengineering, vol. 26, no. 1, pp. $114-119, \quad 2017 . \quad$ Available: 10.13164/re.2017.0114.

[15]Q. Fan, X. Yan and Y. Zhang, "Auto-selection mechanism of differential evolution algorithm variants and its application", European Journal of Operational Research, vol. 270, no. 2, pp. 636-653, 2018. Available: 10.1016/j.ejor.2017.10.013.

[16] T. Li, W. Yan, L. Ping and P. Fang, "A WSN positioning algorithm based on 3D discrete chaotic mapping", EURASIP Journal on Wireless Communications and Networking, vol. 2019, no. 1, 2019. Available: 10.1186/s13638-019-1448-7.

[17]H. Yoshida and Y. Fukuyama, "Parallel Multipopulation Differential Evolutionary Particle Swarm Optimization for Voltage and Reactive Power Control", Electrical Engineering in Japan, vol. 204, no. 3, pp. 31-40, 2018. Available: 10.1002/eej.23100.

[18]X. Zhang , "Population-adaptive differential evolution-based power allocation algorithm for cognitive radio networks", EURASIP Journal on Wireless Communications and Networking, vol. 2016, no. 1, 2016. Available: 10.1186/s13638-016-0722-1. 
[19] T. S. Rappaport, "Wireless Communications Principles and Practice", Pearson Education, $2^{\text {nd }}$ Ed., 1997.

[20] K. Kashyap, M. P. Sarma, K. K. Sarma, and N. Mastorakis, "Generation of Orthogonal Logistic Map Sequences for Application in Wireless Channel and Implementation using a Multiplierless Technique ", $5^{\text {th }} \quad$ International Conference on CIRCUITS,SYSTEMS, ELECTRONICS, CONTROL AND SIGNAL PROCESSING (CSECS '14), Salerno, Italy, Jun. , 2014.

[21] K. Kashyap, K. K. Sarma, M. P. Sarma, and N. Mastorakis , "Generation of Orthogonal Logistic Map Sequences for Faded Channels in Spread Spectrum Modulation" , IEEE International Conference on Signal Processing and Integrated Networks (SPIN 2015), Amity University, Noida, 19$20^{\text {th }}$ Feb. 2015.

[22] K. Kashyap, K. K. Sarma, M. P. Sarma, and N. Mastorakis, "Logistic Map based Spread Spectrum Modulation in Faded Wireless Channel", IEEE International Conference on Signal Processing and Integrated Networks (SPIN 2015), Amity University, Noida, 19-20 ${ }^{\text {th }}$ Feb. 2015.

[23]R. M. May, G. F. Oster. "Chaos from Maps". Journel of Physics Letters A, pp. 1 - 124, vol. 78, Jul. 7, 1980.

[24]P. Biswas, P. Suganthan, R. Mallipeddi and G. Amaratunga, "Optimal power flow solutions using differential evolution algorithm integrated with effective constraint handling techniques", Engineering Applications of Artificial Intelligence, vol. 68, pp. 81-100, 2018

[25] H. Jigang, F. Hui and W. Jie, "A PI controller optimized with modified differential evolution algorithm for speed control of BLDC motor", Automatika, vol. 60, no. 2, pp. 135-148, 2019.

[26] R. Agarwal, H. Sharma and N. Sharma, "Exponential Scale- Factor based Differential Evolution Algorithm", International Conference on Computer, Communications and Electronics, pp. 354-359, 2017.

[27] M. Ali, M. Pant and V. P. Singh, "Two modified differential evolution algorithms and their applications to engineering design problems", World Journal of Modelling and Simulation, vol. 6, no. 1, pp. 72-80, 2010. 